\title{
Inelastic scattering measured on a neutron reflectometer
}

\author{
A.R. Wildes ${ }^{\mathrm{a}}$ \\ Institut Laue-Langevin, 6 rue Jules Horowitz, BP 156, 38042 Grenoble Cedex 9, France
}

Received: 8 August 2011 / Revised: 27 October 2011

Published online: 27 January 2012 - (C) Società Italiana di Fisica / Springer-Verlag 2012

\begin{abstract}
It is possible to use the D17 neutron reflectometer at the Institut Laue-Langevin as a direct geometry time-of-flight spectrometer. The kinematic constraints for the measurement of incoherent scattering and inelastic scattering from highly oriented samples are presented, along with an initial discussion of the energy resolution of the instrument. Inelastic scattering from water and polycarbonate are measured and contrasted with scattering from vanadium. The implications and possibilities for the future measurement of inelastic scattering are discussed.
\end{abstract}

\section{Introduction}

Neutron reflectivity has grown to be one of the most popular neutron scattering techniques, and demand continues to expand. The technique is able to provide valuable and sometimes unique information in a broad range of fields from biology to magnetism. Neutron off-specular scattering, which also may be measured on certain neutron reflectometers, also has great potential and significant efforts are being made to optimize the measurement and analysis methods. The need for reflection geometry instruments is now widely recognized to the point that a reflectometer has featured in the first wave of instruments in several new neutron sources.

Standard practice in reflectometer design and data analysis is to assume that the scattering is essentially elastic. This is not always a good assumption, and some articles have already touched on observable effects in soft [1-3] and hard [4] matter. While these experiments might represent special cases, they raise questions about using a reflectometer to measure inelastic scattering either to discriminate against these effects in the measured data, or to determine directly the dynamics of a material using neutron spectroscopy.

Measuring inelastic scattering requires that the wavelengths of the incident and the scattered beams be determined, and the D17 reflectometer at the Institut Laue-Langevin (ILL), France, is an instrument that is suited for this. It was rebuilt during the late 1990s to be a dedicated neutron reflectometer [5] and was designed with a great deal of flexibility in mind, having both time-of-flight and monochromatic options. If both options are used simultaneously, D17 becomes a direct geometry time-of-flight spectrometer, with the monochromator fixing the incident neutron wavelength and the time of flight giving the final wavelength. Combined with the improved performance of the instrument after a recent upgrade [6], in particular with regards to a new detector, D17 can be used as a quality instrument for measuring inelastic signal at small momentum transfers. This article is a summary of some recent tests that prove the instrument can be used in this mode, along with a discussion of the kinematic constraints of the instrument and of the resolution function.

\section{The D17 instrument}

A schematic of the D17 instrument, with both the choppers and the monochromator in place, is shown in fig. 1. The time-of-flight option makes use of double-disk choppers. This mode of operation has proved to be highly popular, in particular for solid-liquid experiments, and the instrument operates in this mode for $70-80 \%$ of the time. The monochromatic option is almost exclusively used for polarized neutron experiments, as one of the two available monochromators is a polarizing $\mathrm{Fe} / \mathrm{Si}$ periodic multilayer, and is used for the rest of the time. A non-magnetic Ni/Ti periodic multilayer is also available as a monochromator, and is very rarely used. Both monochromators give neutrons of wavelength $\lambda \sim 5.5 \AA$ with a take-off angle of $\sim 4^{\circ}$. They are effectively gradient crystals [7], where the uncertainty

\footnotetext{
${ }^{a}$ e-mail: wildes@ill.fr
} 


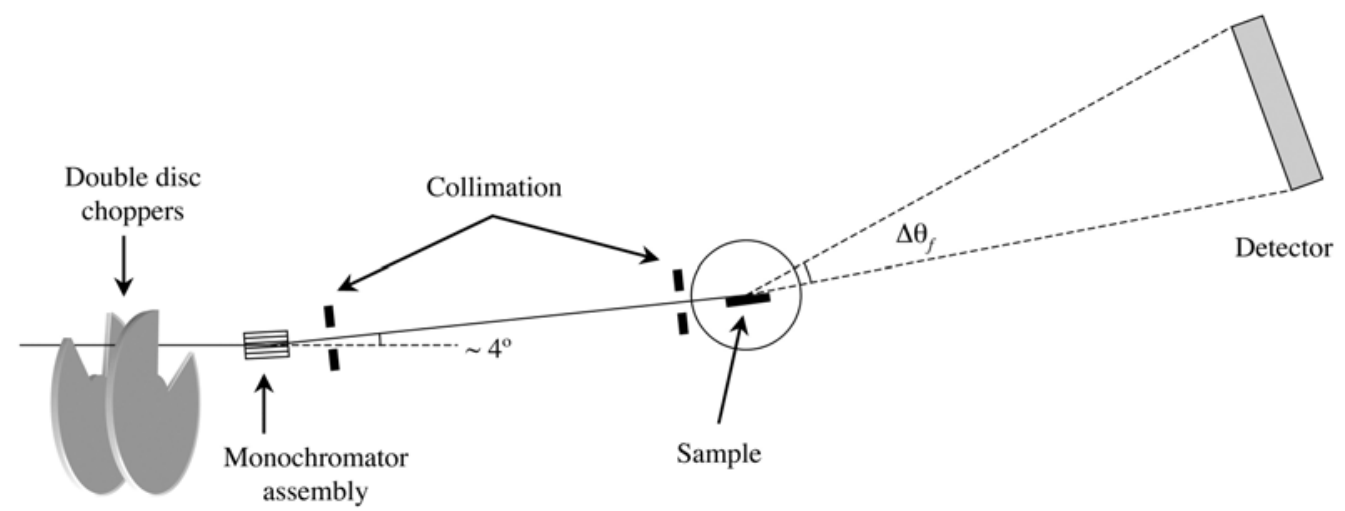

Fig. 1. Schematic showing D17 with both the choppers and monochromator options.

in the incident wavelength is determined by the variation in the d-spacing and not by a mosaic spread. They have been measured using time-of-flight in the instrument to have a wavelength distribution of $\Delta \lambda / \lambda=3 \%$.

The distance from the centre of the choppers to the sample is $D_{i}=4.0815 \mathrm{~m}$ and the separation of the choppers is $D_{c c}=0.087 \mathrm{~m}$. The choppers turn with a period of 0.06 seconds and the wavelength uncertainty is $\Delta \lambda / \lambda=1 \%$ when the chopper openings are phased such that the beam is at the limit of having no direct line-of-sight through them. The distance between the centre of the choppers and the monochromator assembly is $0.2 \mathrm{~m}$. The angular divergence of the incident beam is set by two slits, separated by $3.4 \mathrm{~m}$, that are between the monochromator and the sample. The slits are motorized and their widths may be varied to optimized values for resolution and beam footprint. A "frame overlap" mirror is positioned between the two slits, blocking all neutrons with wavelengths greater than $30 \AA$. The distance from the sample to the detector is variable but was fixed at $D_{f}=3.1 \mathrm{~m}$ for the measurements in this article. At this distance, the position sensitive detector covers an angular range of $\Delta \theta_{f}=4.5^{\circ}$.

The instrument has recently gone through an upgrade which has included a new focusing guide, polarizing optics, and a new position sensitive detector [6]. The new ${ }^{3} \mathrm{He}$ gas detector is made from a single block of aluminium with 64 parallel square holes cut through it. A wire was threaded through each hole to act as a cathode, and amplifiers at either end of each cathode allows a neutron position to be measured to an accuracy of $1.5 \mathrm{~mm}$. The spatial response of the detector is extremely homogeneous, allowing off-specular signals to be measured with unprecedented clarity. Details on the new detector will be given elsewhere [8].

\section{The $(\mathrm{Q}, \hbar \omega)$ manifold}

The following convention will be used throughout this article: bold font variables (e.g., Q) will refer to vectors and italic variables $(e . g ., Q)$ will refer to scalar magnitudes.

The neutron momentum and energy changes, $\mathbf{Q}$ and $\hbar \omega$, are calculated using the equations:

$$
\begin{aligned}
\hbar \omega & =\frac{\hbar^{2}}{2 m_{n}}\left(k_{i}^{2}-k_{f}^{2}\right), \\
\mathbf{Q} & =\mathbf{k}_{i}-\mathbf{k}_{f},
\end{aligned}
$$

where $m_{n}$ is the mass of the neutron, $\hbar$ is the Planck constant divided by $2 \pi$, and $k_{i, f}$ is the wave number of the incident and final beams. If $k_{i, f}$ are given in reciprocal Ångströms and $\hbar \omega$ in meV, the constant $\hbar^{2} /\left(2 m_{n}\right)=2.072 \mathrm{meV} \AA^{2}$.

The instrument measures the total time of flight, $t_{\text {total }}$, from the centre of the choppers to the detector as a function of the scattering angle from the sample, $\theta_{i}+\theta_{f}$. The position sensitive detector sweeps out an angular range, and each pixel on the detector measures a different $\theta_{f}$.

Figure 2 shows a schematic of the regions of the momentum transfer, $\mathbf{Q}$, that are spanned by the instrument when measuring in reflection geometry. The angular limits of the multidetector are shown by the grey lines in the figure. If the initial and final wave vectors, $\mathbf{k}_{i, f}$, are known, the momentum transfer components normal to the surface, $Q_{z}$, and in the plane of the surface, $Q_{x}$, can be calculated using the equations:

$$
\begin{aligned}
Q_{x} & =k_{i} \cos \left(\theta_{i}\right)-k_{f} \cos \left(\theta_{f}\right), \\
Q_{z} & =k_{i} \sin \left(\theta_{i}\right)+k_{f} \sin \left(\theta_{f}\right), \\
Q^{2} & =k_{i}^{2}+k_{f}^{2}-2 k_{i} k_{f} \cos \left(\theta_{i}+\theta_{f}\right) .
\end{aligned}
$$




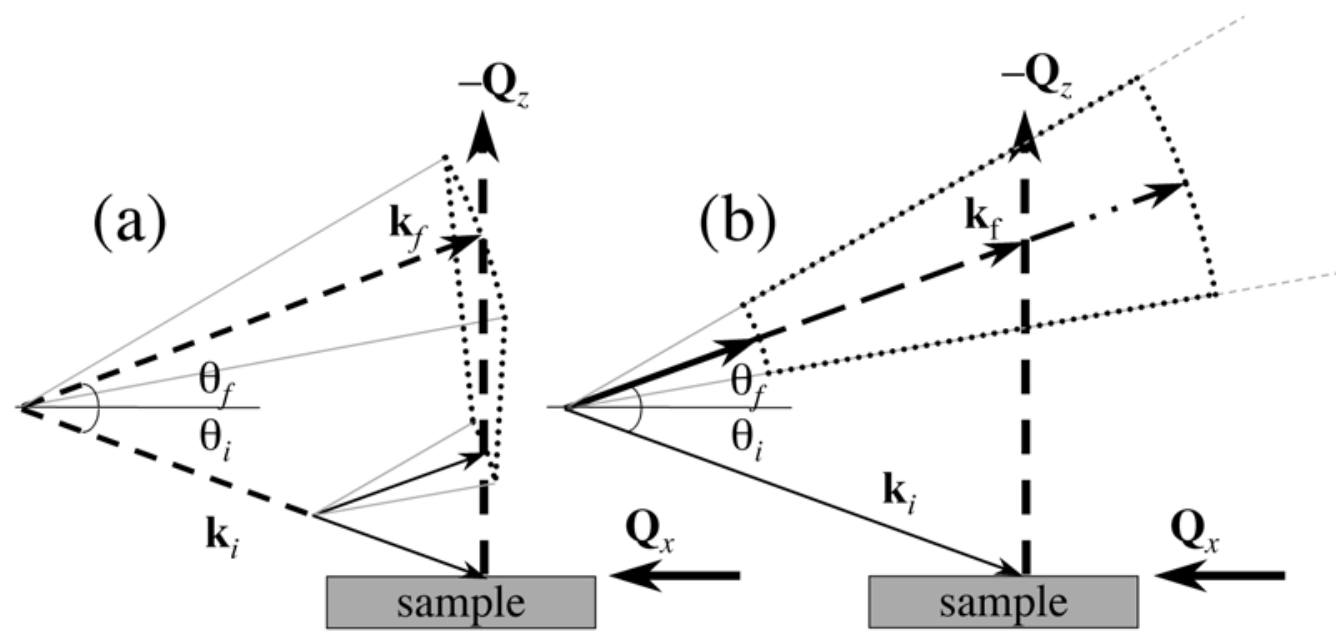

Fig. 2. Schematic showing the $\mathbf{Q}$ manifold when (a) only the choppers are used and the scattering is assumed to be entirely elastic, and (b) both the choppers and the monochromator are used. The grey lines show the $\theta_{f}$ limits set by the width of the multidetector.

While eqs. (2) have been defined relative to the scattering from a surface, similar equations can be defined for smallangle neutron scattering (SANS) instrumentation. The major difference between the two scattering geometries is that reflectometers measure small-angle scattering in grazing incidence from a surface, while SANS instruments measure samples in a transmission geometry. The direction of $\mathbf{Q}_{z}$ is therefore normal to the surface for a reflectometer, but approximately in the plane of the surface for SANS. Small angle approximations for $\theta_{i, f}$ are valid for both geometries.

The time of flight, $t$, for a neutron of wavelength $\lambda$ to cover a distance $D$ is calculated using the equation

$$
t=\frac{m_{n}}{h} D \lambda=\frac{m_{n}}{\hbar} \frac{D}{k}
$$

If $\lambda$ is given in Ångströms, $t$ in seconds and $D$ in metres, the constant $h / m_{n}=3956 \mathrm{~m} \AA \mathrm{s}^{-1}$.

When the choppers are used on their own, it is assumed that the scattering is entirely elastic, i.e. $k_{i}=k_{f}$. The resulting Q-manifold resembles the schematic in fig. 2(a). The specular scattering, defined here as the elastic scattering where $\theta_{i}=\theta_{f}$, is measured in the same few pixels of the detector independently of the wavelength. It is simultaneously measured over a range of $Q_{z}$ thanks to the time of flight. All scattering at $\theta_{i} \neq \theta_{f}$ is considered off-specular.

When both the monochromator and the choppers are used, the incident wavevector $\mathbf{k}_{i}$ is fixed. The time of flight from the choppers to the sample, $t_{i}$, can be calculated using eq. (3), and the time of flight from sample to the detector is used to determine $k_{f}$ via the equation

$$
t_{f}=\frac{m_{n}}{\hbar} \frac{D}{k_{f}}=t_{\text {total }}-t_{i}=t_{\text {total }}-\frac{m_{n}}{\hbar} \frac{D_{i}}{k_{i}} .
$$

The $\mathbf{Q}$ manifold is shown schematically in fig. 2(b). Each $\mathbf{Q}$ now will have a corresponding energy transfer, calculated using eqs. (1). The $\mathbf{Q}$ trajectory swept out for each $k_{f}$ will be an arc centred at the base of $-\mathbf{k}_{f}$ with an angular width given by the size of the multidetector. The specular scattering is now only measured for $\theta_{i}=\theta_{f}$ and a single time-offlight and all the other scattering, irrespective of its angle, is off-specular. Strong off-specular scattering may therefore be due to a the presence of a large amount of inelastic scattering, particular if the scattering is temperature-dependent.

The momentum and energy transfers are heavily coupled. If the scattering is independent of the direction of $\mathbf{Q}$, as is the case for incoherent scattering, the $(Q, \hbar \omega)$ space accessed by the instrument may be calculated with eq. (1) and the last part of eqs. (2). A typical region accessed by D17 is shown in fig. 3. Positive energy transfers correspond to the neutron giving energy to the sample, while negative values corresponding to the neutron receiving energy from the sample. Each of the bold lines represents the $(Q, \hbar \omega)$ trajectory for a pixel at the edge of the detector. The figure shows that, despite the instrument being configured to measure elastic scattering at small $Q$, any inelastic scattering measured will be at significantly larger $Q$. Equation (1) shows that the maximum possible energy that can be given by the neutron to the sample is $\hbar^{2} k_{i}^{2} /\left(2 m_{n}\right)$ while the maximum energy that the neutron can receive is, in principle, $-\infty$.

When measuring samples where directional components of $\mathbf{Q}$ are important, for example from single crystals or, indeed, reflectivity, the momentum and energy transfers become a three-dimensional manifold. Figure 4 shows the manifold of a measurement under the same conditions as fig. 3. The manifold is highly asymmetric. Any constant energy cut through the manifold gives an arc that spans a narrow range in $Q_{x}$, being of order $\sim 10^{-3} \AA^{-1}$, but has 


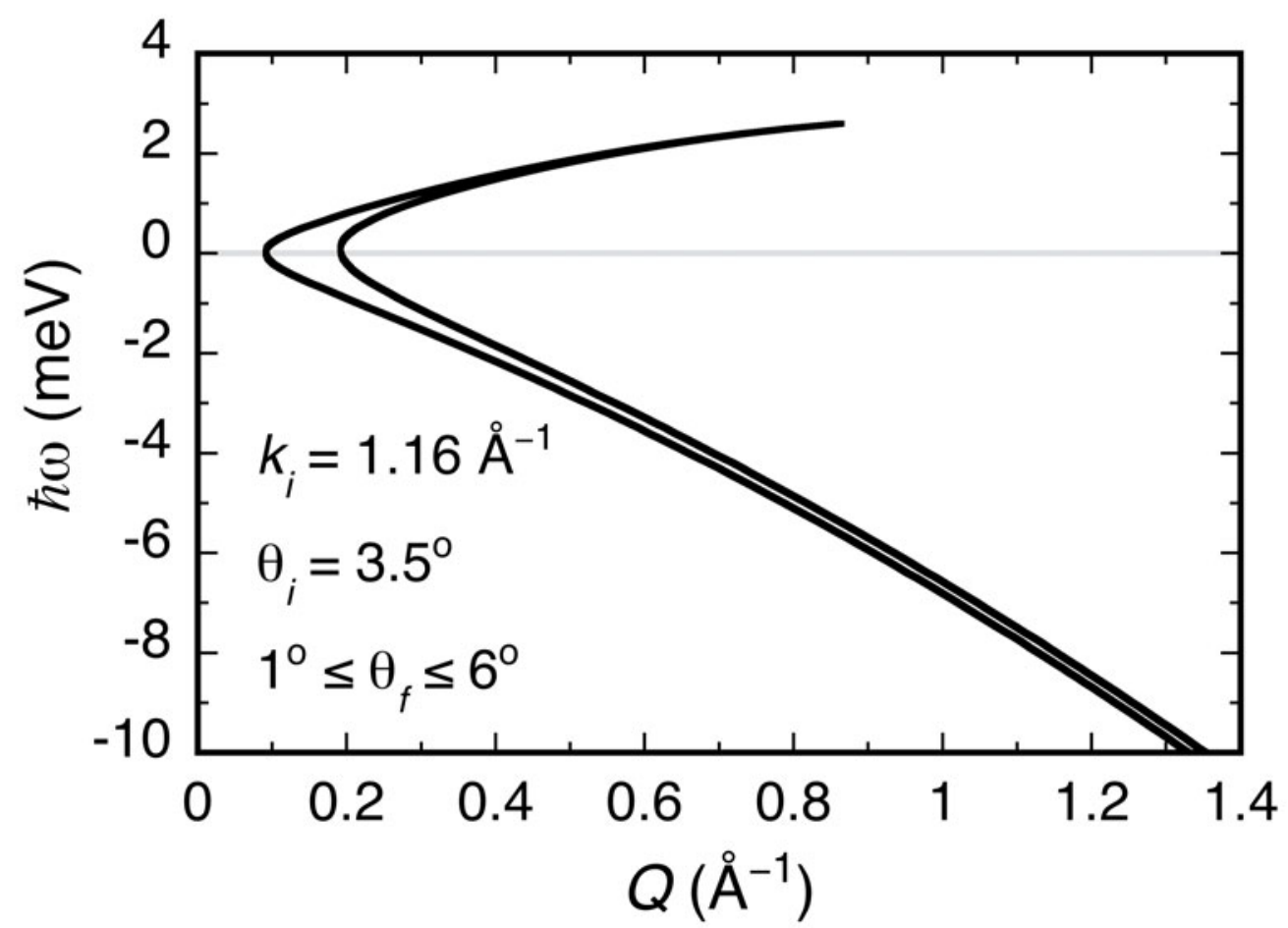

Fig. 3. A calculation giving a typically accessible region of $(Q, \omega)$ that is measured on D17 for one detector position and a fixed $k_{i}$.

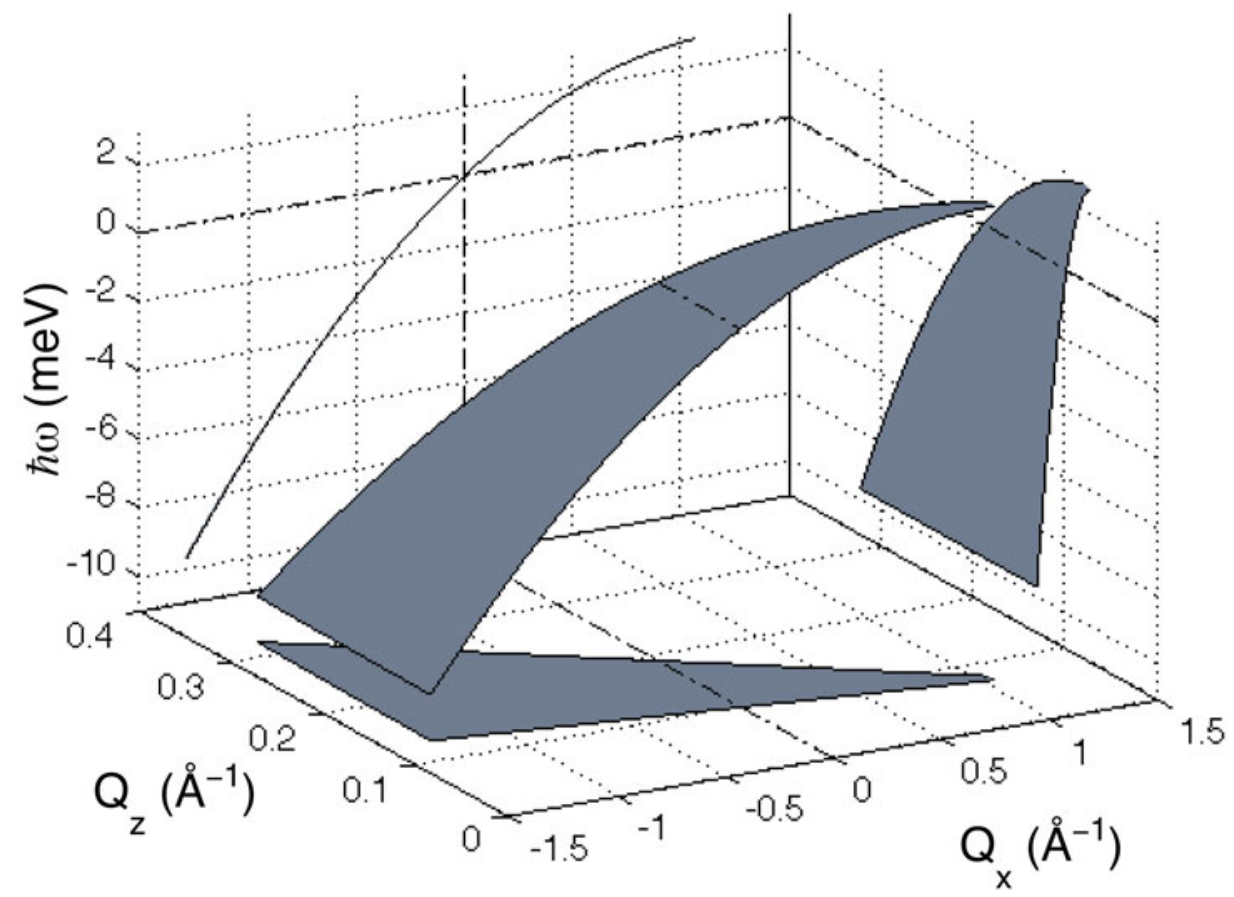

Fig. 4. A typical $(\mathbf{Q}, \hbar \omega)$ manifold that would be measured from a sample in reflection geometry on D17 in inelastic mode, with projections of the manifold on to different planes. The incident wave number and the $\theta_{f}$ range is the same as in fig. 3 . The elastic scattering trajectory is marked on the manifold with a dashed line. 
a range in $Q_{z}$ that is around two orders of magnitude larger. A cut through the manifold at constant $Q_{x}$ gives an arc with an extremely narrow range of energy transfers in the $\left(Q_{z}, \hbar \omega\right)$ plane, being of order $\sim 10^{-3}$ meV. A cut through the manifold at constant $Q_{z}$, however, gives a huge range of momentum and energy transfers, particularly when compared to the small angles and nominal momentum transfers that are used in the measurement. Any measured inelastic signal will have a significant $Q_{x}$ component.

The shape of the manifold clearly limits the types of excitations that can be measured, as scattering will only be observed if the manifold intersects with strong features in the dynamic structure factor. Measurements in reflection geometry are essentially insensitive to excitations traveling along the surface normal, as the accessible energy transfers are extremely small. They will be indistinguishable from elastic scattering as they will be lost within the resolution. Numerous measurements with differing $\theta_{i, f}$ must be made to map out the dynamic structure factor from an oriented sample like a surface. The superposition of numerous manifolds results in the measurement over a volume of $\left(Q_{x}, Q_{z}, \hbar \omega\right)$ space. The angles could be chosen such that the $Q_{x}=0$ trajectory would cut through the manifold at a reasonable energy transfer, being essentially equivalent to a constant energy cut along $Q_{z}$. This cannot be done without either the incident or the scattered beams having to traverse the sample, i.e. the measurement would no longer be in a reflection geometry. The maximum neutron energy transfers that can be achieved at a given $Q_{z}$ and $Q_{x}=0$ while maintaining reflection geometry are when $\theta_{i, f}=0$, i.e. $\hbar \omega= \pm \hbar^{2} Q_{z}^{2} /\left(2 m_{n}\right)$, hence energy transfers at small $Q_{z}$ will always be very small. As an example, measurements at $\left(Q_{x}, Q_{z}\right)=(0,0.2) \AA^{-1}$ with $k_{i}=1.16 \AA^{-1}$ in reflection geometry are limited to energy transfers of $\pm 0.08 \mathrm{meV}$.

On the other hand, inelastic measurements in a reflectivity configuration have potential for studies of excitations traveling in the plane of the sample. The momentum and energy transfers have magnitudes where coherent excitations, such as phonons and magnons, should be measurable. The dispersion surface of these excitations also must intersect with the manifold to give observable scattering, which will limit the range of materials that might be measured with this technique. Close to a Brillouin zone centre, acoustic phonons and magnons in antiferromagnets have excitations whose energies vary linearly with reduced wavelength transfer, $q$. These excitations may be difficult to observe from a Bragg peak which is along the specular direction as the $\left(Q_{x}, \hbar \omega\right)$ gradient of the manifold is also roughly linear around $Q_{x}=0$, hence scattering will only be observed if the gradients roughly match. Magnons from ferromagnets vary with $q^{2}$ at small $q$, however. These may give observable features, particularly if the in-plane ferromagnetic exchange coupling is weak. Low-energy optic modes from both types of excitations should be observable.

The shape of the manifold holds for all neutron reflectometers that use a monochromator to define $k_{i}$. Without energy analysis, though, the values of $\left(Q_{x}, Q_{z}\right)$ are not known and all the scattering for a given $\theta_{f}$ would be integrated.

It is worth noting that excitations traveling along the surface normal could be studied if the sample were rotated such that the surface normal were along $\mathbf{k}_{i}$, as would be the case for a SANS measurement. Clearly the beam would traverse the sample in these types of measurements.

\section{The energy resolution}

The resolution function of the instrument is complicated. It has been previously discussed for elastic scattering on a neutron reflectometer [9], however the strong coupling between momentum and energy transfers at small scattering angles, shown in fig. 3, means that the incident and final beam resolutions cannot be decoupled. This has a strong effect on the resolution which has been discussed for three-axis spectrometry [10,11], but is even further complicated on D17 as eq. (4) shows that the uncertainties in $k_{f}$ and $k_{i}$ are coupled through the time-of-flight method.

The uncertainty in the incident wave number, $\Delta k_{i}$, is given by the gradient monochromator [7] while $\Delta k_{f}$ is given by the width of the time pulse at the sample. For a single wavelength, the time pulse from a double-disk chopper system is given by the equation

$$
\Delta t_{i}=\phi+\frac{m_{n}}{h} D_{c c} \lambda_{i}=\phi+\frac{m_{n}}{\hbar} \frac{D_{c c}}{k_{i}}
$$

where $\phi$ is the time difference between the disk closest to the source closing and the disk closest to the sample opening.

The time width at the sample is broader than that calculated in eq. (5) due to the incident wave number spread. For $\phi=0$, this becomes

$$
\Delta t_{i}{ }^{\prime}=\frac{m_{n}}{\hbar} \frac{D_{c c} k_{i}+D_{i} \Delta k_{i}}{k_{i}^{2}-\frac{1}{4}\left(\Delta k_{i}\right)^{2}} .
$$

The time width of a fully monochromatic beam will remain constant following an energy change at the sample, and the uncertainty in the final wave number will thus be a function of $\Delta t_{i}{ }^{\prime}$, i.e.

$$
\Delta k_{f}=\frac{k_{f}}{t_{f}} \Delta t_{i}{ }^{\prime}=\frac{\hbar}{m_{n}} \frac{k_{f}^{2}}{D_{f}} \Delta t_{i}{ }^{\prime} .
$$


Table 1. Calculations of the energy resolution at different energy transfers on D17 with $k_{i}=1.16 \AA^{-1}$ and the chopper phase time, $\phi$, equal to zero.

\begin{tabular}{ccc}
\hline Energy transfer $(\mathrm{meV})$ & $k_{f}\left(\AA^{-1}\right)$ & $\Delta(\hbar \omega)(\mathrm{meV})$ \\
\hline 1.5 & 0.79 & 0.29 \\
0 & 1.16 & 0.54 \\
-5 & 1.94 & 1.93 \\
-10 & 2.48 & 3.87 \\
\hline
\end{tabular}

The double chopper system delivers a constant $\Delta \lambda / \lambda$ when $\phi=0$, meaning that $\Delta t_{i}{ }^{\prime}$ cannot be adequately described by a Gaussian. This, combined with the fact that the incident and final beams cannot be decoupled, means that resolution convolutions become very difficult to handle. A careful treatment of the resolution function will be forthcoming in a future paper.

For the purposes of this article an extremely simplistic calculation, derived by differentiating eq. (1), may be used. In this manner, the energy resolution may be estimated by

$$
\begin{aligned}
\Delta(\hbar \omega) & =\frac{\hbar^{2}}{2 m_{n}}\left(2 k_{i} \Delta k_{i}+2 k_{f} \Delta k_{f}\right) \\
& =\frac{\hbar^{2}}{2 m_{n}}\left(2 k_{i} \Delta k_{i}+2 \frac{\hbar}{m_{n}} \frac{k_{f}^{3}}{D_{f}} \Delta t^{\prime}{ }^{\prime}\right) .
\end{aligned}
$$

The energy resolution on D17 is rather poor, becoming very poor very quickly in neutron energy gain due to the $k_{f}^{3}$ term in eq. (8). The instrument has a typical $k_{i}=1.16 \AA^{-1}$ and $\Delta k_{i}=0.03 k_{i}=0.035 \AA^{-1}$. With $\phi=0$, eq. (5) gives $\Delta t_{i}=119$ microseconds for the nominal $k_{i}$ and eq. (6) gives $\Delta t_{i}{ }^{\prime}=287$ microseconds for the total time width of the incident beam. These numbers, substituted in to eq. (8), give the resolutions at different energy transfers listed in table 1.

That the energy resolution is poor is unsurprising as the instrument was not optimized as an inelastic spectrometer. The resolution can be improved by over-closing the choppers, corresponding to $\phi<0$, which reduces $\Delta t_{i}^{\prime}$ with a concomitant loss of intensity. Alternatively, the use of a shorter incident wavelength with a similar $\Delta k_{i}$ would mean that larger energy transfers could be measured with neutron energy loss, where the resolution is much better. A wavelength with $2-2.5 \AA$ is possible on D17 and would give excellent flux. However, a new monochromator would need to be installed to access this wavelength given the geometric constraints imposed by the construction of the instrument.

\section{Examples of incoherent scattering}

An incoherent scatterer is often used to calibrate the spatial response of a position sensitive detector. For the reflectometers and SANS instruments at the ILL, a transmission measurement through water is normally used. The scattering from water is known to be highly inelastic, which is unimportant when calibrating the spatial response of a detector but is very important when using it as an intensity standard. It has been suggested that some plastics such as polycarbonate will be better calibration samples [3], although the best is widely acknowledged to be vanadium where the incoherent scattering is overwhelmingly elastic. The inelastic response from both water, polycarbonate, and vanadium were measured on D17.

Figure 5 shows the wavelength spectrum of the direct beam on D17, measured in time of flight without the monochromator. The nominal wavelength may be calculated from the time of flight using eq. $(3)$ with $D=D_{i}+D_{f}$. A measurement of the scattering from a $1 \mathrm{~mm}$ water sample is also shown. For this measurement, the detector was moved to a small scattering angle while making sure that it was not measuring the direct beam. The water distorts the spectrum, shifting it to shorter time of flight, meaning that the neutrons have gained energy from the sample. The nominal wavelength calculation for these data is therefore incorrect.

The degree of the energy change becomes apparent when both the monochromator and the chopper are used. Figure 6 shows two sets of data. The wavelength spectrum of the monochromated beam is shown for both, measured directly in the detector with no sample. The beam was highly collimated and only appears in a few pixels of the position-sensitive detector. The wavelength can, and does in this case, vary slightly from $5.5 \AA$ due to small variations in the instrument alignment. The measurements were made at different times, and the incident wavelength each time was calculated using eq. (3).

The top set of data was measured with the typical time-of-flight conditions used when the detector calibration is carried out on D17, using a relatively large value of $\phi$. The time width of the monochromatic direct beam is 


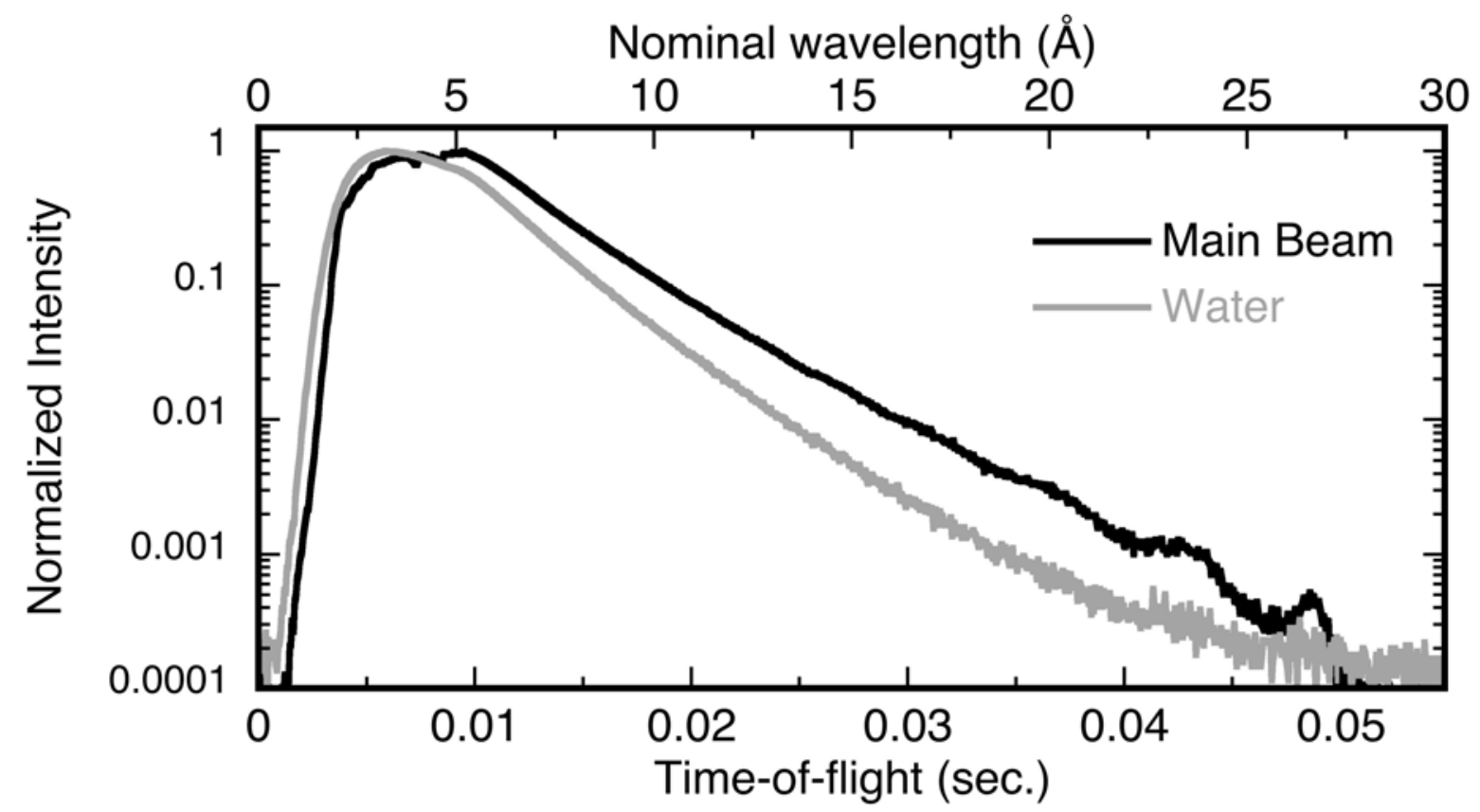

Fig. 5. The main beam spectrum on D17, measured in time of flight with a $\Delta \lambda / \lambda=1 \%$, and the shifted spectrum given by the scattering from water.

consequently also large. The water and polycarbonate measurements were made with the same $\phi$ and the data sets may all be directly compared. The scattering from water and polycarbonate was spread homogeneously across the whole detector and the data in fig. 6 has been integrated over $\theta_{f}$.

The scattering from water has two obvious contributions. Elastic scattering is present, with approximately the same time width as the corresponding direct beam width. There is also a significant inelastic contribution visible at shorter time of flight, corresponding to neutron energy gain. Quasielastic scattering might be present, but is not visible with the resolution used for the measurement.

The inelastic scattering from the water is consistent with previous, similar measurements [3]. The spectral weight of this scattering accounts for $\sim 27 \%$ of the total, and its presence has been previously explained as molecular vibrations [3], including torsional modes at around $55 \mathrm{meV}$. Equivalent spectral weight is not observed at longer times of flight because, as shown by the kinematic constraints of eqs. (1), the incident beam has insufficient energy to excite these modes.

The data are very interesting in their own right as they reveal a number of issues with measuring hydrogencontaining samples, particularly water. Clearly, using water as an intensity calibration, even on cold neutron instruments, is problematic as the scattering is highly inelastic. Intensity measured without energy resolution will thus be highly wavelength- and resolution-dependent, as the instrument will integrate the scattering over some energy range. Putting the intensity on an absolute scale will therefore require a detailed knowledge of the instrument parameters and of the dynamic structure factor, $S(Q, \hbar \omega)$, of the sample so that the energy integration can be correctly made. Effects such as neutron Compton scattering may also be important in water, further complicating the calculation.

Incoherent scattering from hydrogen, and in particular water, is also a major limiting factor in measuring reflectivities and SANS to large $Q$. It is currently very difficult to measure to relative intensities below $10^{-6}$ in SANS from dilute samples and in reflectivity from solid-liquid interfaces as the coherent signal, which falls roughly as $\sim Q^{-4}$, becomes lost in the large sample-dependent incoherent scattering. Figure 6 shows that a significant fraction of the water scattering is inelastic, however. Hence, using some form of energy discrimination for certain measurements may be beneficial to measure coherent scattering at larger $Q$.

Figure 6 also shows a measurement through $1.8 \mathrm{~mm}$ of polycarbonate. The data are qualitatively similar to the water although the spectral weight at large energy transfers is smaller, being $\sim 23.5 \%$ of the total. This makes polycarbonate at room temperature marginally better than water as an intensity calibration standard, however it has an advantage in that it does not dry out and need replenishing. It can also be put in to a cryostat and cooled which will suppress the high neutron energy gain scattering, making it much better as an intensity calibration [3].

For comparison, fig. 6 shows a measurement of the inelastic response in transmission through $1 \mathrm{~mm}$ of vanadium. The data were measured with $\phi=0$, resulting in a much higher time resolution and which allows the wavelength spread from the monochromator to be calculated. The time width of the direct beam spectrum is 450 microseconds. 

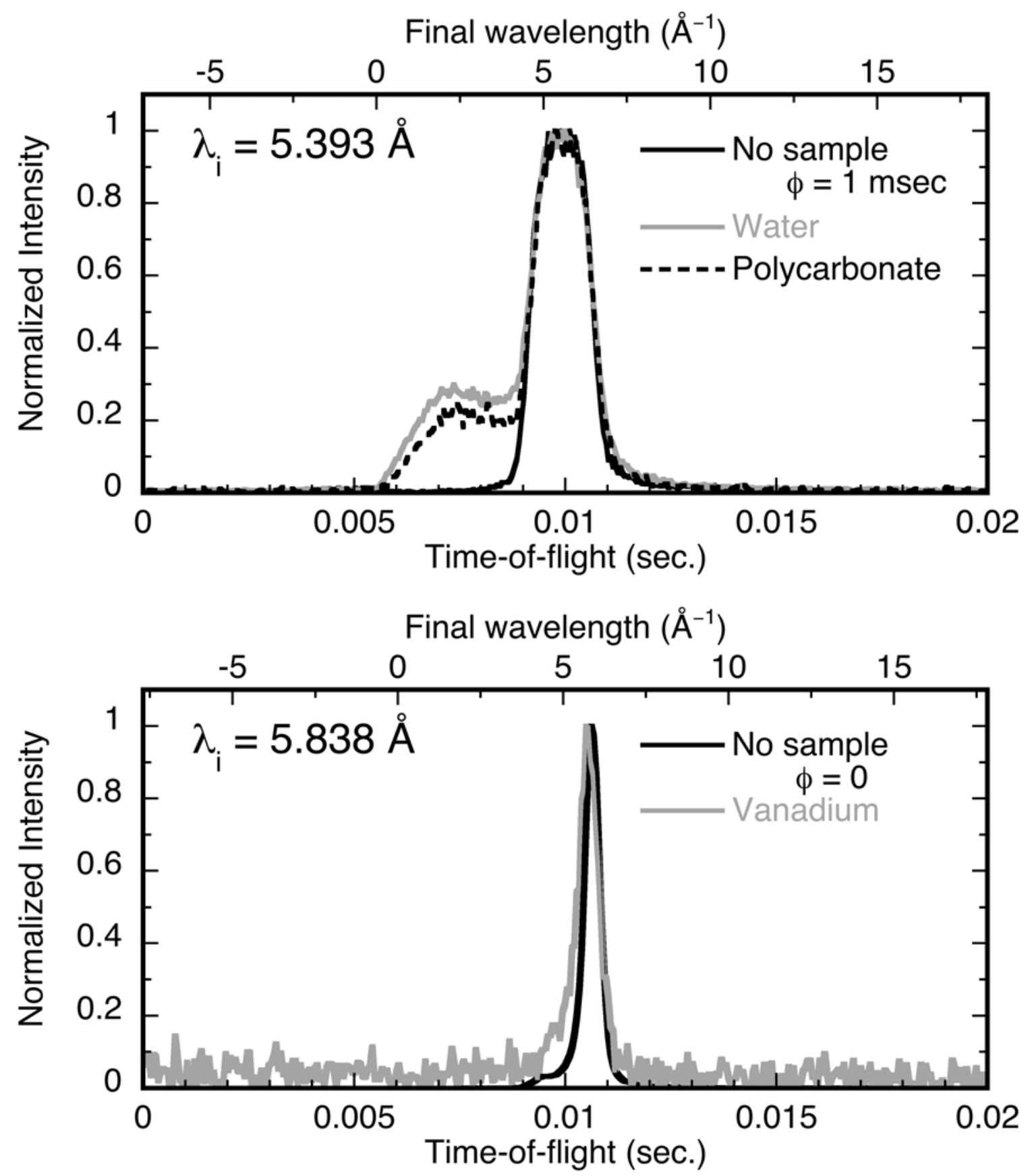

Fig. 6. The spectra of the scattering from water and polycarbonate, measured using on D17, measured with both the monochromator and time of flight in operation. The spectrum of the beam from the monochromator, measured with no sample in the beam, is also presented for two settings of the chopper phase.

Equation (6) may be manipulated to use this time width and the chopper-detector distance to give $\Delta k_{i}=0.03 \AA^{-1}$, which confirms that the intrinsic wavenumber spread is $\Delta k_{i} / k_{i}=3 \%$.

The count rate on the vanadium was about 20 times smaller than that for the water and polycarbonate and the measurement had to be made for much longer to have adequate statistics. The data are therefore noisy, however it can be clearly seen that the vanadium signal is entirely elastic. The vanadium measurement confirms that the energy resolution of the instrument roughly follows the calculations in sect. 4.

The low count rates from this sample mean that collecting adequate statistics to calibrate the detector or the absolute intensity would take a prohibitively long time. The count rate can be boosted by increasing the size of the vanadium, or by increasing $\phi$. However, although it has been useful to check that there are no artifacts in the energy resolution of the instrument, measurements of vanadium are generally unnecessary on D17. The small-angle scattering 
from water is homogeneous, despite the inelasticity, hence measurements of water suffice to calibrate the relative efficiency of the detector. The D17 detector is also capable of measuring the direct beam, and thus cross-sections in absolute units can be calculated by comparing scattered intensities directly with the flux of the incident beam. Both of these measurements are made in the course of normal operation, and would also suffice for any inelastic experiments.

\section{Conclusion}

The neutron reflectometer D17 has been used as a direct geometry inelastic spectrometer to measure incoherent scattering from water, polycarbonate, and vanadium. The measurements confirm the limitations of using incoherent scatterers that have a strong inelastic contribution, such as hydrogen-containing samples, for the intensity calibration of neutron instruments. The instrument can, in principle, be used to measure coherent inelastic scattering in reflection geometry, although the kinematic constraints mean that only in-plane excitations will be measurable. Future work will attempt to measure inelastic scattering in thin films, and derive a comprehensive estimate of the instrument resolution.

The author would like to thank Dr. Bruno Dorner and Dr. Robert Cubitt for constructive discussion and critical reading of the manuscript. Thanks go to the Institut Laue-Langevin for providing the neutron beam time.

\section{References}

1. T. Charitat, S. Lecuyer, G. Fragneto, Biointerphases 3, FB3 (2008).

2. G. Fragneto, M. Rheinstädter, C. R. Phys. 8, 865 (2007).

3. R.E. Ghosh, A. Rennie, J. Appl. Cryst. 32, 1157 (1999).

4. K. Zhernenkov, S. Klimko, B.P. Toperverg, H. Zabel, J. Phys. Conf. Ser. 211, 012016 (2010).

5. R. Cubitt, G. Fragneto, Appl. Phys. A 74, S329 (2002).

6. R. Cubitt et al., ILL newsletter 53, (2010) http://www.ill.eu/quick-links/publications/ill-news/.

7. B. Dorner, Nucl. Instrum. Methods Phys. Res. A 338, 33 (1994).

8. G. Manzin et al., in preparation.

9. B. Dorner, A.R. Wildes, Langmuir 19, 7823 (2003).

10. P.W. Mitchell, R.A. Cowley, S.A. Higgins, Acta Crystallogr. A 40, 152 (1984).

11. H. Grimm, Nucl. Instrum. Methods Phys. Res. 219, 553 (1984). 